\title{
STABLE MANIFOLDS FOR HYPERBOLIC SETS
}

\author{
BY MORRIS W. HIRSCH AND CHARLES C. PUGH
}

Communicated by Jürgen K. Moser, August 20, 1968

1. Introduction. We present a version of the "Generalized stable manifold theorem" of Smale [2, p. 781]. Details will appear in the Proceedings of the American Mathematical Society Summer Institute on Global Analysis.

Let $M$ be a finite dimensional Riemannian manifold, $U \subset M$ an open set and $f: U \rightarrow M$ a $C^{k}$ embedding $\left(k \in Z_{+}\right)$. A set $\Lambda \subset U$ is a hyperbolic set provided

(1) $f(\Lambda)=\Lambda$;

(2) $T_{\Lambda} M$ has a splitting $E^{\mathbb{s}} \oplus E^{u}$ preserved by $D f$;

(3) there exist numbers $C>0$ and $\tau<1$ such that for all $n \in Z_{+}$,

$$
\max \left\{\left\|\left(D f \mid E^{*}\right)^{n}\right\|,\left\|\left(D f \mid E^{u}\right)^{-n}\right\|\right\} \leqq C \tau^{n} .
$$

It is known (J. Mather; see also [1]) that the Riemannian metric on $M$ can be chosen so that $C=1$; we assume $C=1$ in what follows. The splitting is unique.

Notation. If $X$ is a metric space, $B_{r}(x)=\{y \in X \mid d(y, x) \leqq r\}$. If $E$ is a Banach space, $B E=B_{1}(0)$. If $E \rightarrow X$ is a Banach bundle, $B E$ $=U_{x \in X} B E_{x}$.

A submanifold $W \subset M$ is a stable manifold through $x$ of size $\beta$ if $W \cap B_{\beta}(x)$ is closed and consists of all $y \in B_{\beta}(x)$ such that $f^{n}(y)$ is defined and in $B_{\beta} f^{n}(x)$ for all $n \in Z_{+}$.

An unstable manifold is defined to be a stable manifold for $f^{-1}$. Unstable manifolds are easier to handle in proofs, but stable ones are easier to describe notationally. Hence, we confine ourselves to the stable case.

A $C^{k}$ stable manifold system with bundle $E$ is a family of $C^{k}$ submanifolds $\left\{W_{x}\right\}_{x \in \Lambda}$ such that

(4) there exists $\beta>0$ such that each $W_{x}$ is a stable manifold through $x$ of size $\beta$;

(5) $E$ is a vector bundle over $\Lambda$, and there is a map $\phi: V \rightarrow M$ of a neighborhood $V$ of the zero section of $E$ such that $\phi$ maps each $V \cap E_{x}$ diffeomorphically onto $W_{x}$;

(6) $\phi$ is fibrewise $C^{k}$ in this sense: Let $H: A \times R^{q} \rightarrow p^{-1} A$ be a trivialization of $E$ over $A \subset \Lambda$ with $H\left(A \times D^{q}\right) \subset V$. Then each map $\theta_{x}$ $=\phi \circ H \mid x \times D^{a}: D^{a} \rightarrow M$ is $C^{k}$, and $\theta: A \rightarrow C^{k}\left(D^{q}, M\right)$ is continuous. 


\section{Existence and uniqueness.}

TheOREM 1. Let $\Lambda$ be a compact hyperbolic set for $f: U \rightarrow M$. Then there exists a $C^{k}$ stable manifold system $\left\{W_{x}\right\}_{x \in \Lambda}$ with bundle $E^{s}$ such that

(a) each $W_{x}$ is tangent to $E_{x}^{s}$ at $x$;

(b) $\left(W_{x}-\partial W_{x}\right) \cap W_{y}$ is an open (possibly empty) subset of $W_{y}$ for all $x, y$ in $\Lambda$;

(c) there exist numbers $K>0$ and $\lambda<1$ such that if $x \in \Lambda, z \in W_{x}$ and $n \in Z_{+}$then $d\left(f^{n}(x), f^{n}(z)\right) \leqq K \lambda^{n}$.

The proof is based on the following stable manifold theorem for a hyperbolic fixed point in a Banach space. The case $k=1$ is essentially contained in Chapter IX, Lemma 5.1 of Hartman [5].

Let $L(\cdot)$ denote Lipschitz constant.

TheOREM 2. For $i=0,1$ let $T_{i}$ be an invertible linear operator on $a$ Banach space $E_{i}$ such that $\max \left\{\left\|T_{1}^{-1}\right\|,\left\|T_{0}\right\|\right\} \leqq \tau<1$. There exists $\epsilon>0$, depending only on $\tau$, with the following properties. Put $E=E_{0} \times E_{1}$ and $T=T_{0} \times T_{1}$.

(a) If $f: B E \rightarrow E$ satisfies $\max \{|f(0)|, L(f-T)\}<\epsilon$, there exists a unique map $g: B E_{0} \rightarrow B E_{1}$ such that

$$
\text { graph } g=B E \cap f^{-1}(\text { graph } g) \text {; }
$$

(b) $x \in$ graph $g$ if and only if $f^{n}(x) \in B E$ for all $n \geqq 0$;

(c) $L(g) \leqq 1$; and $g$ is $C^{k}$ if $f$ is $C^{k}$, and $g$ depends $C^{k}$ continuously on $f$.

Such a $T$ is a hyperbolic linear map. We call $g$ a stable manifold function for $f$.

Outline of PROOF OF TheOREM 1. Let $\boldsymbol{S}^{b}$ denote the Banach space $S^{b}\left(T_{\Delta} M\right)$ of bounded sections of $T_{\Delta} M$, and $S^{c} \subset S^{b}$ the closed subspace of continuous sections. Let $B^{b}=B \boldsymbol{S}^{b}$ and $B^{c}=B \boldsymbol{S}^{c}$ denote the unit balls. For $x \in \Lambda$ let $e_{x}: M_{x} \rightarrow M$ be the exponential map. If the metric on $M$ is multiplied by a large constant there will be a $C^{k}$ map $f_{b}: B^{b} \rightarrow S^{b}$ given by the formula

$$
f_{b}(\sigma) f(x)=e_{f x}^{-1} f e_{x} \sigma(x) .
$$

(We motivate the definition of $f_{b}$ by considering the Banach manifold $\operatorname{Ir}(\Lambda, M)$ of bounded maps $\Lambda \rightarrow M$ and the local diffeomorphism

$$
F: \operatorname{Tl}(\Lambda, U) \rightarrow \operatorname{Tr}(\Lambda, M)
$$

given by

$$
F(h)=f \circ h \circ\left(f^{-1} \mid \Lambda\right) .
$$


A coordinate chart for $\mathfrak{T}(\Lambda, M)$ with values in $S^{b}\left(T_{\Lambda} M\right)$ is obtained by letting the section $\sigma$ correspond to the map $x \mapsto e_{x} \sigma(x)$ of $\Lambda$ into $M$. The expression for $F$ in these coordinates is then $f_{b}$.) This is the natural action of $f$ on sections $\sigma$.

The derivative of $f_{b}$ at 0 is the hyperbolic linear map $D f_{b}(0) \sigma$ $=D f \circ \sigma \circ f^{-1}$; the corresponding splitting of $S^{b}$ is $S^{b}\left(E^{s}\right) \oplus S^{b}\left(E^{u}\right)$ $=\boldsymbol{S}_{s}^{b} \oplus \boldsymbol{S}_{u}^{b}$. We may assume $L\left(f_{b}-D f_{b}(0)\right)$ so small that by Theorem $1, f_{b}$ has a $C^{k}$ stable manifold function $G^{b}: B_{s}^{b} \rightarrow B_{u}^{b}$ (where $B_{s}^{b}=B S_{s}^{b}$, etc.). Similarly let $G^{c}: B_{s}^{c} \rightarrow B_{u}^{c}$ be the stable manifold function of $f_{c}=f_{b} \mid B^{c}: B^{c} \rightarrow S^{c}$.

Lemma. If $x_{0} \in \Lambda$ and $\sigma_{1}, \sigma_{2} \in B_{s}^{b}$ are such that $\sigma_{1}\left(x_{0}\right)=\sigma_{2}\left(x_{0}\right)$ then $G^{b}\left(\sigma_{1}\right) x_{0}=G^{b}\left(\sigma_{2}\right) x_{0}$.

Proof. $G^{b}(\zeta)$ is the unique section $\xi$ such that $\left|f_{b}^{n}(\zeta x, \xi x)\right| \leqq 1$ for all $n \in Z_{+}$and $x \in \Lambda$, by Theorem 2(b). Applying this to

$$
\begin{aligned}
\zeta_{i}(x) & =0 & & \text { if } x \neq x_{0}, \\
& =\sigma_{i}\left(x_{0}\right) & & \text { if } x=x_{0}
\end{aligned}
$$

proves the lemma.

The lemma implies that $G^{c}=B^{b} \mid B_{g}^{c}$, and that there is a function $H: B E^{s} \rightarrow B E^{u}$ such that $G^{b}(\sigma)=H \circ \sigma$. Also $G^{c}(\sigma)=H \circ \sigma$, implying the continuity of $H$. Each map $H_{x}: B E_{x}^{s} \rightarrow B E_{x}^{u}$ is $C^{k}$. The map $\phi: B E^{s} \rightarrow M$ is defined by $\phi(y)=e(y, H(y))$. It can be shown that $\phi$ is fibrewise $C^{k}$ by writing $\phi$ as the composition.

$$
B E^{s} \stackrel{(\chi, p)}{\longrightarrow} B_{s}^{c} \times \Lambda \stackrel{G^{c} \times 1}{\longrightarrow} B_{u}^{c} \times \Lambda \stackrel{v}{\rightarrow} M
$$

where $p$ is the bundle projection of $E^{s} ; \chi: B E^{s} \rightarrow B_{s}^{c}$ is a fibrewise $C^{k}$ map assigning to each $y \in B E^{s}$ a section of $E^{s}$ through $y$ of norm $\leqq 1$; and $v$ is the evaluation map $v(\sigma, x)=\sigma(x)$.

\section{Smoothness of the splitting of $T_{\Lambda} M$.}

THEOREM 3. Let $f: U \rightarrow M$ be $C^{2}$, and suppose $\Lambda$ is a compact hyperbolic set which is a $C^{2}$ submanifold. Then $E^{*}$ is a $C^{1}$ subbundle of $T_{\Lambda} M$ provided $\left\|D f\left|E^{u}\|\cdot\| D f^{-1}\right| E^{u}\right\| \cdot\left\|D f \mid E^{s}\right\|<1$. In particular this holds if $E^{*}$ has codimension 1 .

The special case $\Lambda=M$ gives

Corollary 4. Let $f$ be a $C^{2}$ Anosov diffeomorphism of a compact manifold $M$. If the stable manifolds have codimension 1 they form a $C^{1}$ foliation of $M$. 
This was stated for $\operatorname{dim} M=2$ in Anosov [4]. On the other hand, Arnold and Avez [3] state that if $E^{s}$ has dimension 1 then the stable manifolds form a $C^{\mathbf{1}}$ foliation.

Outline of PRoOF of Theorem 3. Give $T_{\Lambda} M$ a $C^{1}$ splitting $F^{*} \oplus F^{u}$ approximating $E^{*} \oplus E^{u}$. For each $x \in \Lambda$ the subspace $E_{x}^{*} \subset M_{x}$ is the graph of a linear map $G_{x}: F_{x}^{s} \rightarrow F_{x}^{u}$, and

$$
\text { graph } \left.G_{f x}=D f(x) \text { (graph } G_{x}\right) \text {. }
$$

We consider $G_{x}$ as an element in the bundle $L \rightarrow \Lambda$ whose fibre over $x$ is the Banach space $L_{x}$ of linear maps $F_{x}^{*} \rightarrow F_{x}^{u}$; then $G$ is a section of $L$ invariant under the map $\Gamma: B L \rightarrow B L$ defined as follows. Write $D f^{-1}: F^{\bullet} \oplus F^{u} \rightarrow F^{\bullet} \oplus F^{u}$ as a matrix

$$
\left[\begin{array}{l}
A B \\
C D
\end{array}\right]
$$

where $A: F^{s} \rightarrow F^{s}, B: F^{u} \rightarrow F^{s}, C: F^{s} \rightarrow F^{u}$, and $D: F^{u} \rightarrow F^{u}$ are maps covering $f^{-1}: \Lambda \rightarrow \Lambda$. Define $\Gamma_{x}: L_{x} \rightarrow L_{f-1 x}$ by

$$
\Gamma_{x}(\lambda)=\left(C_{x}+D_{x} \lambda\right) \circ\left(A_{x}+B_{x} \lambda\right)^{-1} .
$$

Theorem 3 is proved once we know that $G$ is $C^{1}$. This follows from

Theorem 5. Let $E \rightarrow M$ be a $C^{1}$ Banach bundle. Let $h: M \rightarrow M$ be a diffeomorphism covered by a $C^{1}$ map $\Gamma: B E \rightarrow B E$. Let $\alpha<1$ be such that each map $\Gamma_{x}: B E_{x} \rightarrow B E_{h x}$ has Lipschitz constant $\leqq \alpha$. Then $B E$ has a unique section $\sigma$ invariant under $\Gamma$. Moreover $\sigma$ is $C^{1}$ provided $\left\|D h^{-1}\right\|$ $<\alpha^{-1}$.

\section{REFERENCES}

1. R. B. Holmes, $A$ formula for the spectral radius of an operator, Amer. Math. Monthly 75 (1968), 163-166

2. S. Smale, Differentiable dynamical systems, Bull. Amer. Math. Soc. 73 (1967), 747-817.

3. V. I. Arnold and A. Avez, Problèmes ergodiques de la mécanique classique, Gauthier-Villars, Paris, 1967.

4. D. V. Anosov, Roughness of geodesic flows on closed Riemannian manifolds of negative curvature, Dokl. Akad. Nauk. SSSR 145 (1962), 707-709=Soviet Math. Dokl. 3 (1962), 1068-1069.

5. P. Hartman, Ordinary differential equations, Wiley, New York, 1964.

University of California, Berkeley, California 94720 